\title{
ALL COUNTABLE GROUPS HAVE CUBIC PRESENTATIONS
}

\author{
MATTHEW A. MARCUS
}

\begin{abstract}
Let $G$ be a group with presentation such that each generator occurs at most countably many times in the set of relations. Then for all $n \geqslant 3, G$ is $n$-ic. In particular, for all $n \geqslant 3$, countable groups have $n$-ic presentations.
\end{abstract}

Neuwirth [1] calls a group $n$-ic if it admits a presentation in which each generator appears exactly $n$ times in the set of relations; such a presentation is also called $n$-ic. He goes on to show that if $G$ is the fundamental group of a closed $n$-manifold then $G * F$ is $n$-ic for some free $F$. Hoare (unpublished; see [2, p. 146]) has shown for $n=3$ that $G$ itself is 3-ic (cubic), and Montesinos [3] extends this result to 3-manifolds with boundary. Here we show that any group that admits a presentation in which each generator occurs at most countably many times in the set of relations is $n$-ic.

The author is indebted to Ken Millett, Marty Scharlemann, and Craig Squier for their invaluable help and encouragement.

We shall employ the following conventions and notation:

Let $X$ be a set and $F$ the free group on $X$. Let $R$ be a set of (reduced) words on $X$ and $\langle\langle R\rangle\rangle_{F}$ the normal closure of $R$ in $F$. Then we say $\langle X \mid R\rangle$ is a presentation for a group $G$ if $G \cong F /\langle\langle R\rangle\rangle_{F}$. In this case we say $G$ is generated by the set $X$ with relations $R$. Now suppose there exist $r_{1}, r_{2} \in R$ such that $w r_{1} w^{-1}=r_{2}^{ \pm 1}$, in $F$, for some $w \in F$. Then $\langle\langle R\rangle\rangle_{F}=\left\langle\left\langle R-\left\{r_{2}\right\}\right\rangle\right\rangle_{F}$, so we may assume that no relation is conjugate to any other relation or its inverse without changing the group. Throughout this paper, 'presentation' means (cyclicly reduced) group presentation with no relation conjugate to any other relation or its inverse.

Notation. Given a presentation $\langle X \mid R\rangle$ with $x \in X$ and $S \subseteq R$, let $\#(x, S)=$ the number of times $x$ occurs in the subset $S$ of relations.

We may now state the main result.

THEOREM. If $G$ is a group with presentation such that each generator appears at most countably many times in the set of relations, then $G$ is cubic.

COROLlaRY. If $G$ is cubic then for all $n \geqslant 3: G$ is $n$-ic.

Received by the editors November 4, 1983 and, in revised form, June 1, 1984.

1980 Mathematics Subject Classification. Primary 20F05: Secondary 57M05.

Key words and phrases. Presentations of groups. 
The proof is based on this rather simple idea: Suppose a generator $x$ appears $n$ times in $R$, where $n$ is finite but larger than three. We modify the presentation in the following manner. First remove $x$ from $X$ and adjoin the formal symbols $x_{1}, \ldots, x_{n}$. Next we remove the relations in which $x$ appears from $R$ and replace them with exactly the same relations except that the $i$ th occurrence of $x$ will be replaced by $x_{i}$ for each $i$. Finally we adjoin the set of relations $\left\{x_{1} x_{2}^{-1}, x_{2} x_{3}^{-1}, \ldots, x_{n-1} x_{n}^{-1}, x_{n} x_{1}^{-1}\right\}$ to $R$. This serves two purposes: it equates all the $x_{i}$ 's so that we still present $G$; and it insures that each $x_{i}$ occurs exactly thrice. We apply a similar series of modifications for each offending generator to obtain the desired presentation.

EXAMPLE. From $\left\langle x, y \mid x^{2} y^{2}, x y x\right\rangle$ we get

$$
\left\langle x_{1}, x_{2}, x_{3}, x_{4}, y \mid x_{1} x_{2} y^{2}, x_{3} y x_{4}, x_{1} x_{2}^{-1}, x_{2} x_{3}^{-1}, x_{3} x_{4}^{-1}, x_{4} x_{1}^{-1}\right\rangle .
$$

Proof of TheOREM. Let $\langle X \mid R\rangle$ be a presentation for a group $G$ satisfying the hypothesis. For $Y \subseteq X$ we define $R_{Y}=R$ with the $k$ th occurrence of each $x \in Y$ replaced by $x_{k}$ for each $k$. Note that $\#(x, R)=\#\left(x, R_{Y}\right)$ if $x \notin Y$, so we may define a set of generators

$$
T_{Y}=\bigcup_{x \in Y}\left\{x_{1}, x_{2}, \ldots, x_{\#\left(x, R_{(Y-\{x\})}\right)}\right\} .
$$

Finally, for each $x \in X$, if we could adjoin a set $S_{x}$ of relations among the $x_{k}$ 's which equate them all and in which each $x_{k}$ appears exactly twice, then

$$
\left\langle\bigcup_{x \in X} T_{x} \mid R_{X} \cup\left(\bigcup_{x \in X} S_{x}\right)\right\rangle
$$

would be a cubic presentation for $G$ since $\#\left(x_{k}, R_{X}\right)=1$ and $\#\left(x_{k}, \cup_{x \in X} S_{x}\right)=$ $\#\left(x_{k}, S_{x}\right)=2$ for each $x \in X$. Unfortunately, we cannot find such $S_{x}$ 's for $\#(x, R)$ $<3$; in this case we must adjoint a few 'dummy generators' $T_{x}^{\prime}$ and include in $S_{x}$ relations amongst elements of $T_{x}^{\prime}$ and the $x_{k}$ 's which equate them all, being carcful to choose $T_{x}^{\prime}$ and $S_{x}$ so that $\#\left(y, S_{x}\right)=3$ for all $y \in T_{x}^{\prime}$ and $\#\left(y, S_{x}\right)=2$ for all $y \in T_{x}$. Then

$$
\left\langle T_{X} \cup\left(\bigcup_{x \in X} T_{x}^{\prime}\right) \mid R_{X} \cup\left(\bigcup_{x \in X} S_{x}\right)\right\rangle
$$

will be our cubic presentation. There are five possible cases:

Case $0 . \#(x, R)=0$.

$$
\begin{aligned}
& T_{x}^{\prime}=\left\{y_{1}, \ldots, y_{4}\right\}, \quad T_{x}=\varnothing, \\
& S_{x}=\left\{y_{1} y_{2}^{-1}, y_{2} y_{3}^{-1}, y_{3} y_{1}^{-1}, y_{1} y_{4}^{-1}, y_{2} y_{4}^{-1}, y_{3} y_{4}^{-1}\right\}, \\
& \forall y \in T_{x}^{\prime}, \#\left(y, S_{x}\right)=3 .
\end{aligned}
$$

Case 1. $\#(x, R)=1$.

$$
\begin{aligned}
& T_{x}^{\prime}=\left\{y_{1}, y_{2}, z_{1}, z_{2}\right\}, \quad T_{x}=\left\{x_{1}\right\}, \\
& S_{x}=\left\{x_{1} z_{1}^{-1}, x_{1} z_{2}^{-1}, z_{1} y_{1}^{-1}, z_{1} y_{2}^{-1}, z_{2} y_{1}^{-1}, z_{2} y_{2}^{-1}, y_{1} y_{2}^{-1}\right\}, \\
& \#\left(x_{1}, S_{x}\right)=2 ; \quad \forall y \in T_{x}^{\prime}, \#\left(y, S_{x}\right)=3 .
\end{aligned}
$$


Case 2. \#(x,R)=2.

$$
\begin{aligned}
& T_{x}^{\prime}=\left\{y_{1}, y_{2}\right\}, \quad T_{x}=\left\{x_{1}, x_{2}\right\}, \\
& S_{x}=\left\{x_{1} y_{1}^{-1}, x_{1} y_{2}^{-1}, x_{2} y_{1}^{-1}, x_{2} y_{2}^{-1}, y_{1} y_{2}^{-1}\right\}, \\
& \forall x_{k} \in T_{x}, \#\left(x_{k}, S_{x}\right)=2 ; \quad \forall y_{k} \in T_{x}^{\prime}, \#\left(y_{k}, S_{x}\right)=3 .
\end{aligned}
$$

Case $3.3 \leqslant \#(x, R)<\infty$.

$$
\begin{aligned}
& T_{x}^{\prime}=\varnothing, \quad T_{x}=\left\{x_{1}, \ldots, x_{n}\right\} \quad \text { where } n=\#(x, R), \\
& S_{x}=\left\{x_{1} x_{2}^{-1}, x_{2} x_{3}^{-1}, \ldots, x_{n-1} x_{n}^{-1}, x_{n} x_{1}^{-1}\right\}, \\
& \forall x_{k} \in T_{x}, \#\left(x_{k}, S_{x}\right)=2 .
\end{aligned}
$$

Case 4. \#(x,R) is countably infinite.

$$
\begin{aligned}
& T_{x}^{\prime}=\varnothing, \quad T_{x}=\left\{x_{1}, x_{2}, \ldots\right\}, \\
& S_{x}=\left\{\ldots, x_{2 i} x_{2 i-2}^{-1}, \ldots, x_{6} x_{4}^{-1}, x_{4} x_{2}^{-1}, x_{2} x_{1}^{-1},\right. \\
& \left.\quad x_{1} x_{3}^{-1}, x_{3} x_{5}^{-1}, \ldots, x_{2 i+1} x_{2 i+3}^{-1}, \ldots\right\}, \\
& \forall x_{k} \in T_{x}, \#\left(x_{k}, S_{x}\right)=2 .
\end{aligned}
$$

In each case note that no two elements of $S_{x}$ are conjugate (in the free group) since each relation has length two, so conjugation either increases length or reverses the order of the elements of each word. Also, $r_{1}, r_{2} \in R_{X}$ implies $r_{1}$ and $r_{2}$ have no letters in common, implying that $r_{1}$ is not conjugate to $r_{2}{ }^{ \pm 1}$ (it was for this reason that we included $\#(x, R)=3$ in the third case). Finally, any conjugate of a word in $S_{x}$ (or its inverse) contains $\cdots a_{i} a_{j}^{-1} \cdots$, where $a_{i}, a_{j} \in T_{x} \cup T_{x}^{\prime}$. But $R_{X}$ cannot contain any word with this configuration, since this implies $R$ contains $x x^{-1}$, contrary to hypothesis.

Proof of Corollary. Suppose $G$ has an $(n-1)$-ic presentation $\langle X \mid R\rangle$. Let $\bar{X}$ be another copy of $X$ whose elements are distinguished from those of $X$ by writing a bar above them. Then $\langle\bar{X} \mid \bar{R}\rangle$ is another $(n-1)$-ic presentation for $G$. Now we equate corresponding elements of $X$ and $\bar{X}$ by adjoining the set of relations $S=\left\{x \bar{X}^{-1} \mid x \in X\right\}$. Note that $\langle X \cup \bar{X} \mid R \cup \bar{R} \cup S\rangle$ is a presentation for $G$, since in the free group on $X \cup \bar{X}$, we have

$$
\begin{aligned}
\langle\langle R\rangle\rangle \cap\langle\langle S\rangle\rangle & =\langle\langle\bar{R}\rangle\rangle \cap\langle\langle S\rangle\rangle=\langle\langle R\rangle\rangle \cap\langle\langle\bar{R}\rangle\rangle \\
& =\text { the empty (identity) word. }
\end{aligned}
$$

Finally, for all $x \in X$,

$$
\#(x, R \cup \bar{R} \cup S)=\#(x, R)+\#(x, \bar{R})+\#(x, S)=(n-1)+0+1=n \text {, }
$$

and similarly for each $\bar{x}$, so in fact we get an $n$-ic presentation for $G$. Using the above theorem, we complete the proof by induction.

\section{REFERENCES}

1. L. Neuwirth, Some algebra for 3-manifolds, Topology of Manifolds (Proc. Inst. Univ. Georgia, 1969), Markham, Chicago, Ill., 1970, pp. 179-184.

2. R. C. Lyndon and P. E. Schupp, Combinatorial group theory, Springer-Verlag, 1977.

3. J. M. Montesinos, Representing 3-manifolds by a universal branching set, Math Proc. Cambridge (to appear).

Department of Mathematics, University of California, Santa Barbara, California 93106

Current address: Department of Mathematics, University of California, Berkeley, California 94720 